This information is published by permission of the Engineer-in-Chief of the Post Office Engineering Department.

P.O. Research Station,

R. BECHMANN

A. C. LYNCH

Dollis Hill, London, N.W.2.

Dec. 30.

${ }^{1}$ Mason, W. P., Phys. Rev., 70, 705 (1946).

${ }^{2}$ Mason, W. P., Proc. Inst. Rad. Eng., 35, 1005 (1947).

${ }^{3}$ Jaffe, H., Phys. Rev., 78, 1467 (1948).

\section{Interferometric Determination of the Apparent Thickness of Thin Metallic Films}

Khamsavi and Donaldson ${ }^{1}$ have described an interferometric method for the measurement of the apparent thicknesses of thin metallic films (200-700 A. in thickness). The method described was essentially that due to Wiener ${ }^{2}$, modified to bring in the ad. vantages of the sharp interference fringes obtained when multiple-beam interferometry is used ${ }^{3}$. An opaque reflecting layer is deposited over the edge of the film to be measured, and the step in this layer is measured by using it as one surface of an interferometer, and viewing this in reflexion using either fringes of equal thickness or fringes of equal chromatic order. By combining these thickness measurements with colorimetric determinations of the mass deposited upon a given area, they showed that the density of the film appeared to remain constant and equal to that of the bulk metal (silver) down to quite small thicknesses (122 A.).

As they themselves pointed out, however, this may be argued to be a necessary result, since the films used probably have small-scale discontinuities in their structure 4 , and the deposition of an opaque reflecting layer over the film to be measured serves to fill up the interstices in that film until its structure resembles that of the opaque film, and hence its density appears to be that of the bulk metal. Some experiments described here tend to confirm this view.

In these experiments a thin film of silver, $A_{1}$ (Fig. 1), was laid down on to a substrate either of silver (previously deposited by evaporation to a thickness of about $1000 \mathrm{~A}$.), or of glass, and over the edge of this, two opaque layers $B_{1}$ and $B_{2}$ were deposited side by side, one of silver and one of some other metal. If the 'packing-in' effect suggested above takes place, it might be expected that the steps in $B_{1}$ and $B_{2}$ would not be of the same height. This has, indeed, been found to be the case. Four typical measurements with the layer $B_{2}$ consisting of chromium, given in the accompanying table, show the magnitude of this effect.

Typical sets of multiple-beam interference fringes of equal thickness running over the steps in $B_{1}$ and

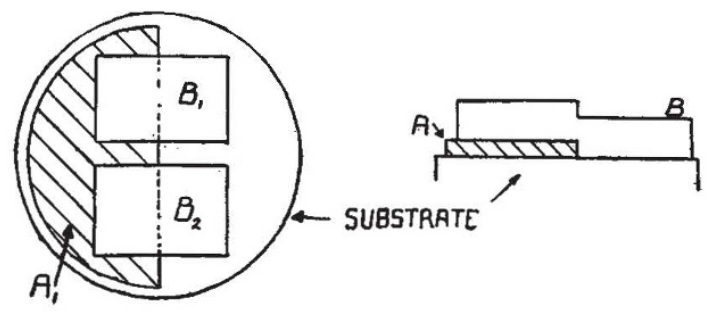

Fig. 1

$\begin{array}{lcccc}\text { Substrate } & \text { Step in } B_{1} & \text { Step in } B_{3} & b & \frac{b}{b_{1}} \times 100 \\ \text { Silver } & 247 & 312 & 65 & 26 \\ \text { Silver } & 470 & 569 & 99 & 21 \\ \text { Glass } & 202 & 279 & 77 & 38 \\ \text { Glass } & 377 & 504 & 127 & 34\end{array}$

$B_{2}$ are shown in Fig. 2. Owing to the relatively low reflectivity of the chromium layers, the fringes obtained over $B_{2}$ are very broad, and the accuracy of the measurements throughout is not greater than $\pm 25 \mathrm{~A}$.

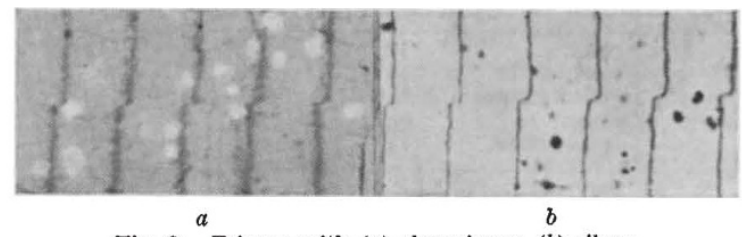

Fig. 2. Fringes with (a) chromium; $(b)$ silver

These results show that measurements of the thinner film thicknesses obtained by this method should be treated with caution, since errors of the order of 30 per cent at least may occur, possibly greater. The limitations of the method should therefore be recognized. It should be noted, however, that the method of Khamsavi and Donaldson, because of the constancy found for the apparent density, affords an accurate means of estimating the mass deposited per unit area in a given film, provided that the layer deposited over the edge of the film is of the same metal as the film itself.

I wish to express my thanks to Prof. S. Tolansky, under whose direction this work was carried out, and to the Department of Scientific and Industrial Research for provision of a maintenance grant.

\section{Royal Holloway College,}

Englefield Green, Surrey. Dec. 28.

'Khamsavi and Donaldson, Nature, 159, 228 (1947).

Wiener, Wied. Ann., 31, 629 (1887).

"Tolansky, "Applications of Multiple Beam Interferometry" (Oxford Univ. Press, 1948).

4 Thomson. Proc. Phys. Soc., 61, 403 (1948).

\section{Absorption Spectra, Molecular Weights, and Visual Purple}

Houston ${ }^{1}$ has shown that, in the case of absorbing media, the number $p$ of electrons per molecule of solute giving rise to an absorption band with a maximum at the wave-length $\gamma_{m}$ is given by

$$
p=\frac{4 \pi n k_{m}}{s} \cdot \frac{m}{e} \cdot \frac{m_{H}}{e} \cdot \frac{\lambda^{\prime}-\gamma_{m}}{\gamma_{m}{ }^{3}},
$$

where $e$ and $m$ are the electronic charge and mass. respectively, $m_{\boldsymbol{H}}$ the mass of the hydrogen atom, $n$ the refractive index of the solution at ${ }_{m}, k_{m}$ the absorption coefficient at $?_{m}, s$ the concentration in grammolecules per c.c., and $\lambda^{\prime}$ the wave-length at which the absorption index is half the value $k_{m}$. Houston calculated $p$ for some thirty substances, and found values between 0.1 and 1 in eighteen cases; between 1 and 2.7 in four cases; and considerably less than unity in the remaining cases.

In other words, in the great majority of solutions $p$ is about unity or less. This fact offers a new method for the estimation of the molecular weight of substances having an absorption band in the visible or ultra-violet part of the spectrum, for, if we assume $p=1$, equation (1) becomes on rearrangement 